\title{
Automatic Human Fall Detection Using Multiple Tri-axial Accelerometers
}

\author{
${ }^{1}$ Fouzi Harrou \\ ${ }^{1}$ King Abdullah University of Science \\ and Technology (KAUST) \\ CEMSE Division \\ Thuwal, Saudi Arabia \\ ${ }^{1}$ Ying Sun \\ ${ }^{1}$ King Abdullah University of Science \\ and Technology (KAUST) \\ CEMSE Division \\ Thuwal, Saudi Arabia
}

\author{
${ }^{2,3}$ Nabil Zerrouki \\ ${ }^{2}$ Center for Development \\ of Advanced Technologies, DIIM Team \\ Baba Hassen, Algeria
}

\author{
${ }^{4}$ Abdelkader Dairi \\ ${ }^{4}$ University of Science and Technology of \\ Oran-Mohamed Boudiaf, SIMPA Laboratory, \\ Oran, Algeria
}

\author{
${ }^{3}$ Amrane Houacine \\ ${ }^{3}$ University of Sciences and Technology Houari Boumédienne, \\ LCPTS, Faculty of Electronics and Computer Science \\ Algiers, Algeria
}

\begin{abstract}
Accurately detecting human falls of elderly people at an early stage is vital for providing early alert and avoid serious injury. Towards this purpose, multiple triaxial accelerometers data has been used to uncover falls based on an unsupervised monitoring procedure. Specifically, this paper introduces a oneclass support vector machine (OCSVM) scheme into human fall detection. The main motivation behind the use of OCSVM is that it is a distribution-free learning model and can separate nonlinear features in an unsupervised way need for labeled data. The proposed OCSVM scheme was evaluated on fall detection databases from the University of Rzeszow's. Three other promising classification algorithms, Mean shift, ExpectationMaximization, k-means, were also assessed based on the same datasets. Their detection performances were compared with those obtained by the OCSVM algorithm. The results showed that the OCSVM scheme outperformed the other methods.
\end{abstract}

\section{INTRODUCTION}

Accurate gesture recognition leads to a better understanding of human behavior [1], [2]. This paper presents an innovative approach to uncover falls based on multiple triaxial accelerometers data. This approach uses one class algorithm for accurate and reliable detection of fall incidents. Essentially, the One Class Support Vector Machine (OCSVM) method is applied to identify features corresponding to fall signals. The major benefit of using OCSVM is its capacity in separating nonlinear features. Furthermore, unlike several methods that are based on training on sequences from both classes namely: daily activity and fall incidents, OCSVM involves a training phase using only fall-free data and then reject fall incident with labeling it as an outlier. The effectiveness of this approach has been evaluated using the fall detection databases from the University of Rzeszow's. Results demonstrated superior fall detection performances of OCSVM compared to Mean shift, Expectation-Maximization, k-means algorithms.

It is worth understanding that there is no perfect approach that in any situation induces the most accurate detections [3], [4], [5]. All of these formalisms have their advantages and disadvantages. In the first class of approaches, the proposed systems have been focused on the processing of data acquired by living environment sensors [6], [7], [8], [9], such as pressure sensors, floor vibration sensors, ultrasonic sensor networks, or acoustic sensors [10]. The main shortcoming of these solutions is the need to place different sensors within the environment in which the elder lives. Furthermore, these approaches based on environmental sensors strongly depend on devices being placed at mostly fixed locations, which make detection limited only on areas equipped with sensors. In the case of acoustic-based sensors, the detection interfered with sounds or noises in the living environment, which could be subject to false alarms. A comparable disadvantage is also encountered in the case of vibration sensors where fall incident is usually confused with other activities like jumps or heavy walking.

Otherwise, vision-based fall detection approaches have been widely used during the last few years where various systems have been explored, including single charge-coupled device cameras [11], stereo-pair cameras [12], and multiple cameras [13]. The study in [14] employed the learned features from video sequences and applied a detection threshold to distinguish between fall incidents from everyday activities. The detection threshold has been fixed manually. Nevertheless, this procedure leads to a high false detection rate, and the threshold value impacted the final performance. Authors in [15] considered fall detection in a home care system. Specifically, they applied the Directed Acyclic Graph SVM approach to detect falls based on ellipse approximation and horizontal and vertical projection histograms. In [16], a vision-driven technique has been introduced for detecting multi-occupancy fall based on convolutional neural networks. In [17] a vision method based on a neural network is employed to detect fall situations. In [8] , authors used k-nearest neighbor (KNN) to recognize fall events. This simple approach reached a suitable detection 
accuracy of about $95 \%$ in detecting falls. In [18], a three-step approach is presented to detect falls using multi-camera fusion. After specifying the number of considered cameras, every camera decides individually regarding the fall incident, and a final decision is taken by combing decisions from every single camera using an aggregation function. Recently, in [19], authors proposed a vision-driven technique to detect human falls based on Spatial-Temporal Graph Convolutional Networks. They highlighted that this approach could be implemented using new data with no need for retraining the model. The use of the surveillance camera seems to be the definitive solution for the detection of human falls due to the important number of proposed methods in the literature. However, the camera-based solution is limited to spaces under video monitoring, which cannot attain an omnipresent observation and many people think it affects elderly people's privacy.

The last class of fall detection systems employs wearable devices with integrated Micro-Electro-Mechanical Systems (MEMS) such as several motion sensors like accelerometers or gyroscopes in order to identify fall incidents and generate an alarm [20], [21]. In fact, fall incidents correspond to sudden changes in sensors' measurements. Changes in acceleration during a fall are identified by setting thresholds on sensor outputs, and magnitudes exceeding the defined thresholds are then declared as falls [8]. However, the threshold value is fixed manually and empirically where it is based on repeated tests which cause a considerable number of false alarms. Inspired by airbag systems applied for car crashes, Purwar et al. [22] used a tri-axial accelerometer by calculating trunk's angle, angle acceleration, and set their control limits to detect falls. In the same context, Bourke et al. [23] used a bi-axial gyroscope and defined three thresholds applied on resultant angular velocity, resultant angular acceleration, and the resultant change in trunk-angle. The authors tested this approach on a total data set of 480 movements, and they obtained very satisfactory results. In [24], authors have been empirically studied the feasibility of using smartwatches in wearable fall detection systems. In [25], a simple Recurrent Neural Networks classifier based on two Long Short-Term Memory (LSTM) cells has been investigated in identifying human falls. The Softmax is used as the output layer. The SisFall dataset data is used to verify the performance of this approach, which comprises sequences of tri-axial accelerometer and gyroscope readings. Results reveal reasonable classification performance of RNN-based classifier.

In previous works [26], the authors combined accelerometer and camera-based strategy to develop human fall detection and human action separation. Several anomaly detection strategies have been applied to sensor data one can cite: exponentially weighted moving average (EWMA) [26], and multivariate exponentially weighted moving average (MEWMA) control chart [27]. Past studies on fall accidents of the elders reported that acceleration sensor-based approaches cannot provide sufficient data, thus can only provide judgment with limited conditions, and cannot provide correct data of falling stances and impacted parts [28]. In addition, fall incidents do not follow a specific model, from which it is so hard to apply a given training on the fall sequences. The objective of this study is to detect unusual events, especially fall events, between free fall videos. The main idea behind the choice of OCSVM classifier is its capacity to use only positive information corresponding to fall-free sequences (including all usual activities) and then detect abnormal events, especially fall incidents as outliers. In other words, OCSVM tries to classify one class of all daily activities, and should then be trained to reject each fall incident and label it as an outlier.

The remainder of this paper is structured as follows. Section II describes accelerometer features and their time evolution. In Section IV, we briefly describe the OCSVM classification technique used to distinguish fall incidents from all daily activities. In Section V, we present experimental results. Finally, we offer conclusions in Section VI.

\section{FEATURES GENERATION}

In this paper, the row accelerometer data have been used as characteristic coefficients. The three accelerometer values namely: $\mathrm{A}_{x}, \mathrm{~A}_{y}$, and $\mathrm{A}_{z}$ which reflect the acceleration measurements along the $\mathrm{x}, \mathrm{y}$, and $\mathrm{z}$ axes, respectively.

These accelerometer components are presented in gravity units $(\mathrm{g})$. The main idea behind the use of accelerometer components as attributes in fall detection is their sensibility and their appropriate characterization of fall accidents. One can note that the pre-fall instant, which is supposed to still represent daily activities, is generally characterized by some signs of instability. Furthermore, the impact instant is characterized by a vertical shock when the body hits the ground corresponding to the formation of the main peaks in the accelerometer components. In some cases, this main peak can be followed by a series of attenuated peaks related to other minor impacts.

In addition to these three gravity components, a total Sum Vector $\mathrm{SV}_{\text {Total }}$ is also calculated and used as another feature. For each triplet of accelerometer data, the total sum vector is calculated as follows:

$$
S V_{\text {Total }}=\sqrt{A_{x}^{2}+A_{y}{ }^{2}+A_{z}^{2}}
$$

$\mathrm{SV}_{\text {Total }}$ is then concatenated with the three accelerometer components to define the whole feature vector which will be used as input in the classification phase.

\section{OCSVM CLASSIFICATION:}

The objective of this classification method is to train the OCSVM only on positive information, corresponding to the magnitude of acceleration components from the fall-free data (including all daily and normal activities) and then detect abnormal events, especially fall incidents.

The particularity of the 1SVM scheme resides in its training with only anomaly-free observations to learn nominal behavior 
in the investigated process, and no labeling is needed to construct 1SVM. Crucially, 1SVM is an unsupervised binary classifier [29]. 1SVM aims to determine a hyper-plan as far as possible from the origin; mainly, the hyper-plan should be as close as possible to the normal observation of the training data points in the original space [30]. Then, it is used to classify new testing data as comparable or different from the training data. ISVM has been widely employed for anomaly detection in different applications.

Let $\left\{x_{1}, x_{2}, \ldots, x_{l}\right\}, x_{i} \in X \in \mathcal{R}^{d}$ be the training set, where $l \in \mathcal{N}$ is the number of observations that belong to a single class, daily activities in our case. The principal of OCSVM classifier is to define the boundary of $\Gamma$, the minimum volume region enclosing $(1-\gamma) l$ observations. Hyperparameter $\gamma, \in[0 ; 1]$, controls the fraction of observations that are allowed to be out of $\gamma$ (outliers, fall incidents in our case). Let $f_{X}$ a decision function such that:

$$
\left\{\begin{array}{l}
f_{x}(x) \geq 0 \quad \text { if } \mathrm{x} \in \Gamma \\
f_{x}(x)<0 \quad \text { otherwise }
\end{array}\right.
$$

Within SVM, the space of possible functions $f_{x}(X)$ is reduced to a Reproducing Kernel Space, called feature space.

The aim when training OCSVM is to define the separation hyperplane $W=\{h \in H$ s.t.hh, wiH $-b=0\}$, so that the margin $\frac{b}{\|w\|_{H}}$ is maximum, where parameters $w$ and $b$ denote the solutions of the following minimization problem.

$$
\begin{gathered}
\min _{\mathrm{w}, \xi, \mathrm{b}} \frac{1}{2}\|\mathrm{w}\|_{H}^{2}-b+\frac{1}{v \mathrm{l}} \sum_{i=1}^{l} \xi_{i} \\
\left\{\begin{array}{c}
<\mathrm{w}, \varphi\left(x_{i}\right)>_{H} \geq \mathrm{b}-\xi_{i} \\
\xi_{i} \leq 0, i=1 \ldots l
\end{array}\right.
\end{gathered}
$$

where $\xi_{i}$ denote the slack variables taht represent the loss associated with (non-null $\xi_{i}$ define the outliers). The Lagrangian multipliers $\alpha$ associated with this problem fully determine $w$ and $b$. The decision function is expressed as: $f_{x}(x)=\sum_{i} \alpha_{i} k\left(x_{i}, x\right)-b$ and $\left\{x_{j} / a_{j} \neq 0\right\}$ represents the support vectors.

\section{EXPERIMENTS}

In this section, we report on the effectiveness of the proposed OCSVM approach in detecting fall incidents. Toward this end, we performed experiments on real and publicly available datasets: URFD [8]. The interdisciplinary Centre provides this database for Computational Modelling, which includes seventy (thirty falls + forty activities of daily living (ADL)) sequences corresponding to the camera and accelerometer data. The data has been measured using PS Move $(60 \mathrm{~Hz})$ and $\mathrm{x}$-IMU $(256 \mathrm{~Hz})$ sensors. The $\mathrm{x}$-IMU device permits to provide measurements of the acceleration along the $x, y$, and $\mathrm{z}$-axes. The accelerometer measures the direction of gravity and provides an absolute reference for the pitch and roll components of the estimated orientation. The $\mathrm{x}$-IMU device has a 12-bit resolution and selectable ranges from $\pm 2 \mathrm{~g}$ to \pm 8 $\mathrm{g}$, as illustrated in Figure 1.

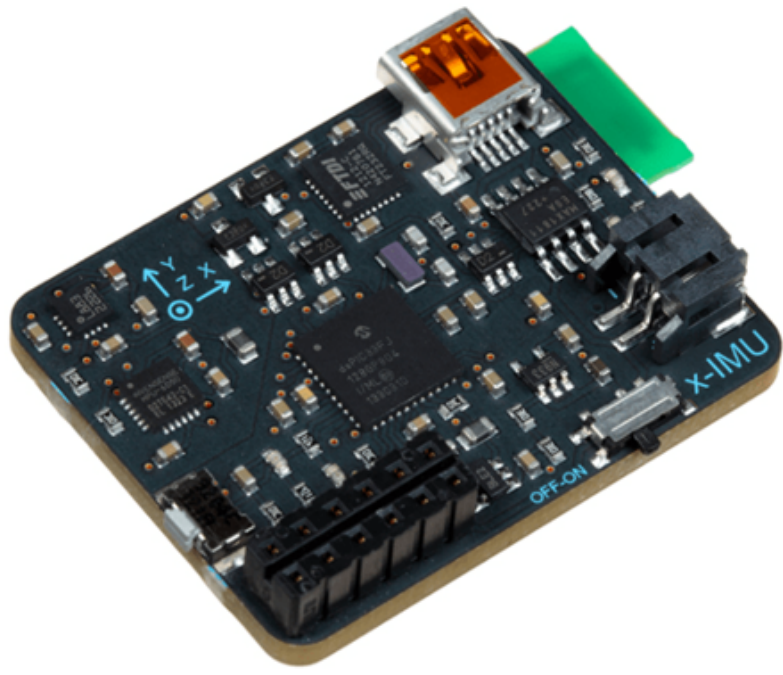

Fig. 1. x-IMU sensor used in accelerometer data measurement.

In the case of the URFD database, accelerometer data related to each ADL or fall incident contains time in milliseconds (since sequence start) and the corresponding accelerometer data, namely: SVtotal, Ax, Az, and Ay, which are all in gravity units $(\mathrm{g})$. This dataset represents a challenging task due to diverse activities, including walking, picking up an object from the ground, changing positions like walking, sitting or sitting, and getting up. Furthermore, URFD Benchmark is challenging due to the presence of activities similar to fall incidents, such as intentional lying, which are considered in the ground truth as ADL sequences.

\section{A. Description of each example}

Here, we present some examples of different daily activities or fall incidents investigated in this work (Figure 2). The time evolution of tri-axial acceleration components and SVtotal corresponding to daily activities like picking up an object from the floor, sitting down standing up are in Figure 2(a), while that correspond to fall events are illustrated in Figure 2 (b-c). Figure 2(a) shows acceleration components in the first scenario while the monitored person performs different daily activities (no falls). One can notice that the corresponding accelerometer magnitudes remain relatively smalls and do not exceed 2.5 g. On the other hand, when a fall occurs, the accelerometer magnitudes increase significantly, signalizing the occurrence of a new situation that is importantly distinguishable from the activities of daily living (Figure 2(b-c)) where accelerometer magnitudes surpassed the value of $7 \mathrm{~g}$.

As mentioned in section II, the pre-fall instant is usually characterized by some signs of instability (Figure 2 (b-c)), while the impact moment is characterized by the main peak corresponding to the moment when the body hits the ground. In some situations, the main peak can be followed by a series of minor peaks (Figure 2(c)). 

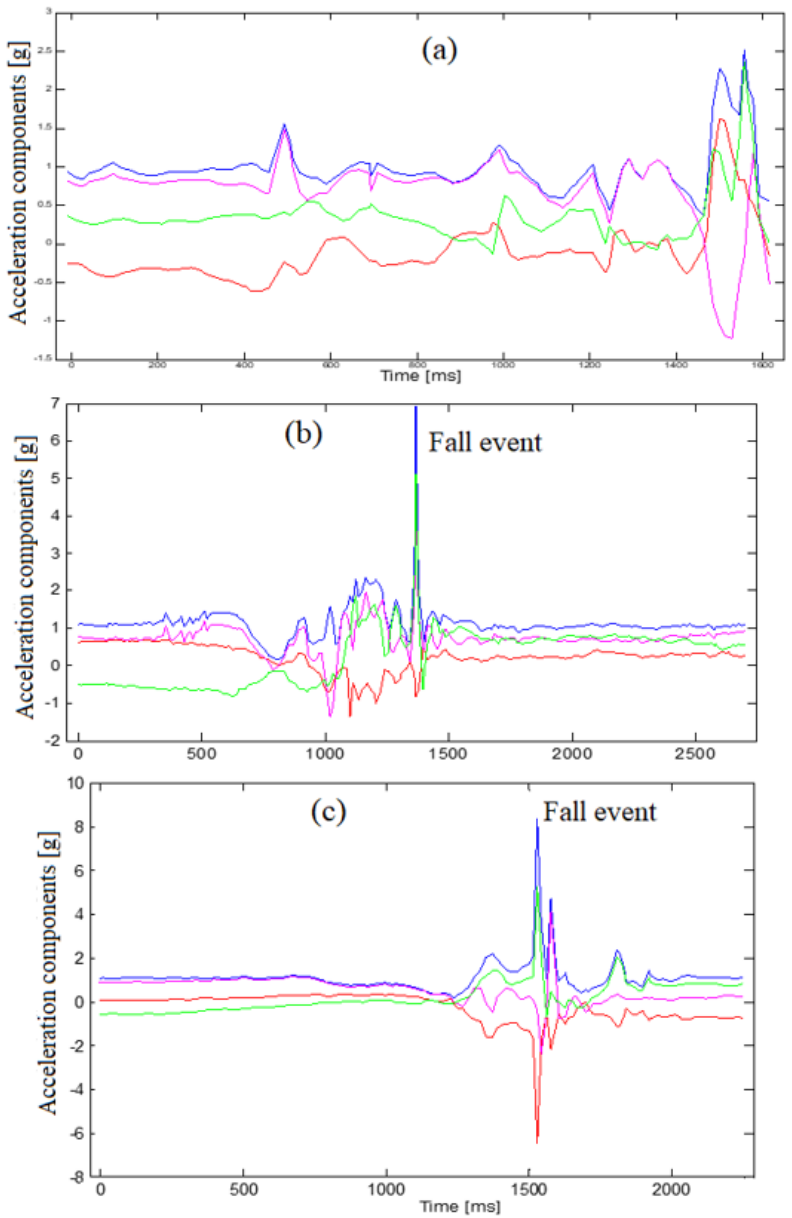

Fig. 2. Time evolution of acceleration components in case of (a) daily activities, (b) and (c) fall event situations.

\section{B. Result and interpretation}

Table 1 summarizes the detection performance when applying four machine-learning techniques to the URFD datasets in terms of the overall accuracy F-measure and area under curve area (AUC).

TABLE I

EVALUATION OF THE INVESTIGATED FALL DETECTION PROCEDURES.

\begin{tabular}{|c|c|c|c|c|}
\hline Classifier & $\begin{array}{c}\text { Accuracy } \\
(\%)\end{array}$ & Precesion & $\begin{array}{c}\text { F- } \\
\text { measure }\end{array}$ & AUC \\
\hline Mean Shift & 80.9 & 80.4 & 0.67 & 0.801 \\
\hline $\begin{array}{c}\text { Expectation } \\
\text { Maximisation }\end{array}$ & 76.3 & 58.6 & 0.488 & 0.664 \\
\hline K-means & 62.3 & 62.17 & 0.438 & 0.578 \\
\hline OCSVM & $\mathbf{9 3 . 7}$ & $\mathbf{7 1 . 4}$ & $\mathbf{0 . 7 8}$ & $\mathbf{0 . 9 1 3}$ \\
\hline
\end{tabular}

The results in Table 1 demonstrate that the OCSVM procedure achieves superior detection performance compared to the
Mean shift, Expectation-Maximization, k-means algorithms. Indeed, OCSVM provides the highest accuracy, which shows its capacity to distinguish real falls. In other words, the number of false negatives (FNs) or false falls is reduced, where only rare like-fall cases were confused as true falls. The reason that the OCSVM algorithm outperformed the other powerful classifiers like K-means, Mean shift, and Mean shift, is that OCSVM has a different strategy in detecting falls, where the training is applied only on free-fall data or normal activities, while fall cases are automatically rejected and labeled as outliers. Unlike the other classifiers, the model is trained on both classes: normal activities and fall incidents. It is worth establishing those fall scenarios are generally dissimilar, making their corresponding data complex to be modeled.

In summary, the OCSVM procedure provides a promising way to discriminate daily activities from fall events, outperforming other competitors. An important feature of OCSVM is its capability for ensuring a globally optimal solution for a given training data. Another important feature is OCSVM's capability to operate as a kernel-based machine learning algorithm, thus separating nonlinear features without using labeled data. The overall detection performance of the OCSVMbased fall detection scheme based on the accelerometer was satisfying.

\section{CONClusion}

Accurate gesture recognition leads to a better understanding of human behavior. This paper presented an innovative approach to uncover falls based on multiple triaxial accelerometers data. This approach uses one class algorithm for accurate and reliable detection of fall incidents. Essentially, the One Class Support Vector Machine (OCSVM) method is applied to identify features corresponding to fall signals. The major benefit of using OCSVM is its capacity in separating nonlinear features. Furthermore, unlike several methods that are based on training on sequences from both classes namely: daily activity and fall incidents, OCSVM involves a training phase using only fall-free data and then reject fall incident with labeling it as an outlier. The effectiveness of this approach has been evaluated using the fall detection databases from the University of Rzeszow's. Results demonstrated superior fall detection performances of OCSVM compared to Mean shift, Expectation-Maximization, k-means algorithms.

To further improve the detection capacity of the proposed approach, in future work, we plan to use more input data from smartphones and smartwatches, which are easy to collect and permit free body movement for the seniors. Essentially, it is planned to consider more information from data inputs including blood pressure and heart rate gathered via smartwatch or a smartphone in order to improve the efficiency of a fall detection system 


\section{ACKNOWLEDGMENT}

This publication is based upon work supported by King Abdullah University of Science and Technology (KAUST), Office of Sponsored Research (OSR) under Award No: OSR-2019-CRG7-3800.

\section{REFERENCES}

[1] W. H. Organization, W. H. O. Ageing, and L. C. Unit, WHO global report on falls prevention in older age. World Health Organization, 2008.

[2] P. Pierleoni, A. Belli, L. Palma, M. Pellegrini, L. Pernini, and S. Valenti, "A high reliability wearable device for elderly fall detection," IEEE Sensors Journal, vol. 15, no. 8, pp. 4544-4553, 2015.

[3] J. Chen, K. Kwong, D. Chang, J. Luk, and R. Bajcsy, "Wearable sensors for reliable fall detection," in 2005 IEEE Engineering in Medicine and Biology 27th Annual Conference. IEEE, 2006, pp. 3551-3554.

[4] N. Noury, P. Rumeau, A. Bourke, G. ÓLaighin, and J. Lundy, "A proposal for the classification and evaluation of fall detectors," Irbm, vol. 29, no. 6, pp. 340-349, 2008.

[5] L. Tong, Q. Song, Y. Ge, and M. Liu, "Hmm-based human fall detection and prediction method using tri-axial accelerometer," IEEE Sensors Journal, vol. 13, no. 5, pp. 1849-1856, 2013.

[6] G. Shi, C.-S. Chan, Y. Luo, G. Zhang, W. J. Li, P. H. Leong, and K.-S. Leung, "Development of a human airbag system for fall protection using mems motion sensing technology," in 2006 IEEE/RSJ International Conference on Intelligent Robots and Systems. IEEE, 2006, pp. 44054410.

[7] G. Shi, C. S. Chan, W. J. Li, K.-S. Leung, Y. Zou, and Y. Jin, "Mobile human airbag system for fall protection using mems sensors and embedded svm classifier," IEEE Sensors Journal, vol. 9, no. 5, pp. 495-503, 2009.

[8] B. Kwolek and M. Kepski, "Improving fall detection by the use of depth sensor and accelerometer," Neurocomputing, vol. 168, pp. 637645, 2015.

[9] S. Nooruddin, M. M. Islam, F. A. Sharna, H. Alhetari, and M. N. Kabir, "Sensor-based fall detection systems: a review," Journal of Ambient Intelligence and Humanized Computing, pp. 1-17, 2021.

[10] X. Zhuang, J. Huang, G. Potamianos, and M. Hasegawa-Johnson, "Acoustic fall detection using gaussian mixture models and gmm supervectors," in 2009 IEEE International Conference on Acoustics, Speech and Signal Processing. IEEE, 2009, pp. 69-72.

[11] D. Anderson, J. M. Keller, M. Skubic, X. Chen, and Z. He, "Recognizing falls from silhouettes," in 2006 International Conference of the IEEE Engineering in Medicine and Biology Society. IEEE, 2006, pp. 63886391.

[12] B. Jansen and R. Deklerck, "Context aware inactivity recognition for visual fall detection," in 2006 Pervasive Health Conference and Workshops. IEEE, 2006, pp. 1-4.

[13] R. Cucchiara, A. Prati, and R. Vezzani, "A multi-camera vision system for fall detection and alarm generation," Expert Systems, vol. 24, no. 5, pp. 334-345, 2007.

[14] C. Rougier and J. Meunier, "3d head trajectory using a single camera," in International Conference on Image and Signal Processing. Springer, 2010, pp. 505-512.

[15] M. Yu, A. Rhuma, S. M. Naqvi, L. Wang, and J. Chambers, "A posture recognition-based fall detection system for monitoring an elderly person in a smart home environment," IEEE transactions on information technology in biomedicine, vol. 16, no. 6, pp. 1274-1286, 2012.

[16] J. M. Quero, M. Burns, M. A. Razzaq, C. Nugent, and M. Espinilla, "Detection of falls from non-invasive thermal vision sensors using convolutional neural networks," in Multidisciplinary Digital Publishing Institute Proceedings, vol. 2, no. 19, 2018, p. 1236.

[17] L. Alhimale, H. Zedan, and A. Al-Bayatti, "The implementation of an intelligent and video-based fall detection system using a neural network," Applied Soft Computing, vol. 18, pp. 59-69, 2014.

[18] S. Ezatzadeh, M. R. Keyvanpour, and S. V. Shojaedini, "A human fall detection framework based on multi-camera fusion," Journal of Experimental \& Theoretical Artificial Intelligence, pp. 1-20, 2021.

[19] O. Keskes and R. Noumeir, "Vision-based fall detection using st-gen," IEEE Access, vol. 9, pp. 28 224-28 236, 2021.

[20] J.-S. Lee and H.-H. Tseng, "Development of an enhanced thresholdbased fall detection system using smartphones with built-in accelerometers," IEEE Sensors Journal, vol. 19, no. 18, pp. 8293-8302, 2019.
[21] L. Chen, R. Li, H. Zhang, L. Tian, and N. Chen, "Intelligent fall detection method based on accelerometer data from a wrist-worn smart watch," Measurement, vol. 140, pp. 215-226, 2019.

[22] A. Purwar, D. U. Jeong, and W. Y. Chung, "Activity monitoring from real-time triaxial accelerometer data using sensor network," in 2007 International conference on control, automation and systems. IEEE, 2007, pp. 2402-2406.

[23] A. K. Bourke and G. M. Lyons, "A threshold-based fall-detection algorithm using a bi-axial gyroscope sensor," Medical engineering \& physics, vol. 30, no. 1, pp. 84-90, 2008.

[24] F. J. González-Cañete and E. Casilari, "A feasibility study of the use of smartwatches in wearable fall detection systems," Sensors, vol. 21, no. 6, p. 2254, 2021.

[25] R. Shrivastava and M. Pandey, "Ensemble of multiple classifiers for accelerometer based human fall detection," in Computer Networks and Inventive Communication Technologies. Springer, 2021, pp. 865-874.

[26] N. Zerrouki, F. Harrou, Y. Sun, and A. Houacine, "Accelerometer and camera-based strategy for improved human fall detection," Journal of medical systems, vol. 40, no. 12, pp. 1-16, 2016.

[27] F. Harrou, N. Zerrouki, Y. Sun, and A. Houacine, "A simple strategy for fall events detection," in 2016 IEEE 14th International Conference on Industrial Informatics (INDIN). IEEE, 2016, pp. 332-336.

[28] Harrou, Fouzi and Zerrouki, Nabil and Sun, Ying and Houacine, Amrane, "Statistical control chart and neural network classification for improving human fall detection," in 2016 8th International Conference on Modelling, Identification and Control (ICMIC). IEEE, 2016, pp. 1060-1064.

[29] F. Harrou, Y. Sun, A. S. Hering, M. Madakyaru, and A. Dairi, "Unsupervised deep learning-based process monitoring methods," in Statistical Process Monitoring Using Advanced Data-Driven and Deep Learning Approaches. Elsevier, 2021, pp. 193-223.

[30] B. Schölkopf, J. C. Platt, J. Shawe-Taylor, A. J. Smola, and R. C. Williamson, "Estimating the support of a high-dimensional distribution," Neural computation, vol. 13, no. 7, pp. 1443-1471, 2001. 